\title{
ON PROJECTIVE MODULES OVER FINITE QUANTUM GROUPS
}

\author{
CRISTIAN VAY* \\ Universidad Nacional de Córdoba \\ Facultad de \\ Matemática, Astronomía \\ Física y Computación \\ CIEM - CONICET \\ Córdoba, Argentina \\ vay@famaf.unc.edu.ar
}

\begin{abstract}
Let $\mathcal{D}$ be the Drinfeld double of the bosonization $\mathfrak{B}(V) \# \mathbb{k} G$ of a finitedimensional Nichols algebra $\mathfrak{B}(V)$ over a finite group $G$. It is known that the simple $\mathcal{D}$-modules are parametrized by the simple modules over $\mathcal{D}(G)$, the Drinfeld double of $G$. This parametrization can be obtained by considering the head $\mathrm{L}(\lambda)$ of the Verma module $\mathrm{M}(\lambda)$ for every simple $\mathcal{D}(G)$-module $\lambda$. In the present work, we show that the projective $\mathcal{D}$-modules are filtered by Verma modules and the BGG Reciprocity $[\mathrm{P}(\mu): \mathrm{M}(\lambda)]=$ $[\mathrm{M}(\lambda): \mathrm{L}(\mu)]$ holds for the projective cover $\mathrm{P}(\mu)$ of $\mathrm{L}(\mu)$. We use graded characters to prove the BGG Reciprocity and obtain a graded version of it. As a by-product we show that a Verma module is simple if and only if it is projective. We also describe the tensor product between projective modules.
\end{abstract}

\section{Introduction}

The representation theory of the universal enveloping algebras of Lie algebras has plenty of powerful and beautiful methods and results which have served as inspiration to the study of modules over many other algebras. The first property that was extended to other contexts is that the simple modules are in bijective correspondence with the simple modules of the Cartan subalgebra. This was made by several authors for the Drinfeld double $\mathcal{D}$ of the bosonization $\mathfrak{B}(V) \# \mathbb{k} G$ of a finite-dimensional Nichols algebra $\mathfrak{B}(V)$ over a finite group $G$; see for instance [8], [5], [31], [18] for $G$ abelian, and [23], [24], [28] for general $G$. In these works $\mathcal{D}(G)$, the Drinfeld double of the underlying group, plays the role of the Cartan subalgebra. Namely, let $\Lambda$ be a complete set of non-isomorphic simple $\mathcal{D}(G)$ modules and $\mathrm{M}(\lambda)$ denote the generalized Verma module of $\lambda \in \Lambda$. Then, the head $\mathrm{L}(\lambda)$ of $\mathrm{M}(\lambda)$ is simple and every simple $\mathcal{D}$-module can be obtained in this way, see loc. cit.

DOI: $10.1007 /$ s00031-017-9469-y

*Partially supported by CONICET, Secyt (UNC), FONCyT PICT 2016-3957, Programa de Cooperación MINCyT-FWO and MathAmSud project GR2HOPF.

Received December 29, 2016. Accepted September 24, 2017.

Corresponding Author: C. Vay, e-mail: vay@famaf.unc.edu.ar 


\section{CRISTIAN VAY}

Here we continue this approach and investigate the projective modules over $\mathcal{D}$. We obtain the following results.

(I) Every projective $\mathcal{D}$-module $\mathrm{P}$ has a (graded) standard filtration.

That is, there is a sequence of (graded) submodules $0=\mathrm{N}_{0} \subset \mathrm{N}_{1} \subset \cdots \subset \mathrm{N}_{n}=\mathrm{P}$ such that each $N_{i} / N_{i-1}$ is isomorphic to a Verma module. We denote by $[P: M(\lambda)]$ the numbers of subquotients isomorphic to the Verma module $M(\lambda), \lambda \in \Lambda$.

(II) The BGG Reciprocity holds in our setting.

More precisely, let $\mathrm{P}(\mu)$ be the projective cover of $\mathrm{L}(\mu), \mu \in \Lambda$ (it exists because $\mathcal{D}$ is a finite-dimensional algebra over an algebraically closed field $\mathbb{k}$ of characteristic zero). Then, for all $\lambda \in \Lambda$,

$$
[\mathrm{P}(\mu): \mathrm{M}(\lambda)]=[\mathrm{M}(\lambda): \mathrm{L}(\mu)]
$$

where the last square brackets denote the numbers of occurrences of $\mathrm{L}(\mu)$ as composition factor of $\mathrm{M}(\lambda)$.

We also give analogous results to (I) and (II) for co-Verma modules. As consequence, we show that

(III) A Verma module is simple if and only if it is projective.

(IV) The tensor product between projective modules is isomorphic to the induced module from a semisimple $\mathcal{D}(G)$-module.

The BGG Reciprocity has its origin in the work of Bernstein-Gelfand-Gelfand [10] for modules in the category $\mathcal{O}$. To achieve our goals we imitate the strategy in [19, Sect. 3] developed by Irving [20], who has also defined axiomatically a class of highest weight categories for which the BGG Reciprocity holds [21]. However, our algebra $\mathcal{D}$ does not completely fit in these frameworks. Indeed, a highest weight category has finite global dimension but a finite-dimensional non-semisimple Hopf algebra is Frobenius and then it has infinite global dimension.

A more general definition of highest weight category was given by Kleshchev [22]. Although this can have infinite global dimension, there must exist a partial order $\leq$ on $\Lambda$ such that $\mu \leq \lambda$ if $\mathrm{L}(\mu)$ is a composition factor of the Verma module $\mathrm{M}(\lambda)$, as in the definition of Cline-Parshall-Scott [13]. This property may fail in our case. For instance, if $\mathfrak{B}(V)$ is the Fomin-Kirillov algebra $\mathcal{F K}_{3}$ and $G$ is the symmetric group $\mathbb{S}_{3}$, we have shown that the composition factors of $\mathrm{M}(\tau, 0)$ and $\mathrm{M}(e, \rho)$ are the same: $\mathrm{L}(\tau, 0), \mathrm{L}(\sigma,-)$ and $\mathrm{L}(e, \rho)[28$, Thms. 9 and 10]. Then, such an order on $\Lambda$ will imply that $(\tau, 0)=(e, \rho)$, a contradiction. The order also ensures that the simple modules are identified by their characters but the characters of $\mathrm{L}(\tau, 0)$ and $\mathrm{L}(e, \rho)$ are equal [28, Cors. 22 and 24].

After this work appeared, Bellamy and Thiel [9] introduced a highest weight theory for finite-dimensional graded algebras with triangular decomposition. They show that the category of graded modules over such an algebra is highest weight. They noted that $\mathcal{D}$ fits in their framework [9, Sect. 8.5] and hence some of our results can be deduced. As $\mathcal{D}$ is a finite-dimensional Hopf algebra, there are some peculiarities in this setting which are not present in [9] but are instrumental in our proofs. For instance, (a) the dual of a Verma module is isomorphic to a Verma module and (b) the tensor product between a Verma module and a co-Verma module is the induced module from a semisimple $\mathcal{D}(G)$-module (Lemma 4 ). 
Let us summarize the main ideas in the present work. First, we observe that the Nichols algebra $\mathfrak{B}(V)$ is finite-dimensional and graded. Thus, we can consider graded characters that allow us to distinguish the simple modules (Theorem 9) since they are characterized by their highest-weights. The finiteness assumption is useful to lead to the standard filtrations of projective modules (Theorem 15) using (b). It also implies that the homogeneous component of maximal degree in $\mathfrak{B}(V)$ is one-dimensional. We use this fact to prove that the set of Verma modules is closed by taking duals (see (10)). Then, we find some identities among the graded characters of a Verma module, its dual and a co-Verma module (Theorem 10). As a consequence we give a graded version of the BGG Reciprocity similar to [22, Thm. 7.6]. More explicitly, for all $\lambda, \mu \in \Lambda$ there exist Laurent polynomials $p_{\mathrm{P}(\mu), \mathrm{M}(\lambda)}, p_{\mathrm{M}(\lambda), \mathrm{L}(\mu)} \in \mathbb{Z}_{\geq 0}\left[t, t^{-1}\right]$ such that the graded characters of $\mathrm{P}(\mu)$ and $\mathrm{M}(\lambda)$ satisfy

$$
\operatorname{ch}^{\bullet} \mathrm{P}(\mu)=\sum_{\lambda \in \Lambda} p_{\mathrm{P}(\mu), \mathrm{M}(\lambda)} \operatorname{ch}^{\bullet} \mathrm{M}(\lambda) \quad \text { and } \quad \operatorname{ch}^{\bullet} \mathrm{M}(\lambda)=\sum_{\mu \in \Lambda} p_{\mathrm{M}(\lambda), \mathrm{L}(\mu)} \operatorname{ch}^{\bullet} \mathrm{L}(\mu)
$$

in the ring $\mathbb{Z} \Lambda\left[t, t^{-1}\right]$. Then, we show in Corollary 12 that

$$
p_{\mathrm{P}(\mu), \mathrm{M}(\lambda)}=\overline{p_{\mathrm{M}(\lambda), \mathrm{L}(\mu)}}
$$

in the ring $\mathbb{Z}\left[t, t^{-1}\right]$, where the ring automorphism $\overline{(\mathrm{)}}: \mathbb{Z}\left[t, t^{-1}\right] \rightarrow \mathbb{Z}\left[t, t^{-1}\right]$ interchanges $t$ and $t^{-1}$. By evaluating these polynomials at $t=t^{-1}=1$ we obtain the BGG Reciprocity (Theorem 15).

Finally, we point out some by-products of our results. Let us assume that we know the graded characters of the simple modules. Then, we can deduce the graded structure of the indecomposable projective modules from the graded version of the BGG Reciprocity. Moreover, we can infer the tensor product between simple and projective modules from the multiplication of their graded characters in the ring $\mathbb{Z} \Lambda\left[t, t^{-1}\right]$. We carry out this plan for $\mathfrak{B}(V)=\mathcal{F} \mathcal{K}_{3}$ and $G=\mathbb{S}_{3}$ jointly with Barbara Pogorelsky in [29].

The article is organized as follows. We set our conventions and notations in Section 2, and state immediate properties of the $\mathcal{D}$-modules. We consider the graded characters in Section 3. The results (I)-(IV) are proved in Section 4. We give some examples in Section 5.

Acknowledgments. I thank N. Andruskiewitsch for the interesting and guiding discussions, and his comments which helped me improve this work. Also, I want to thank I. Angiono and V. Ostrik for the useful conversations. Part of this work was carried out during a visit to the University of Antwerp. I am grateful to F. Van Oystayen and Y. Zhang for their warm hospitality and interesting discussions. Finally, I thank the referees for the careful reading of the manuscript and for pointing out the reference [16] for Lemma 5 .

\section{Preliminaries}

We assume that the reader is familiar with Hopf algebras and Nichols algebras. For a survey on Nichols algebras we refer to [6]. 


\section{CRISTIAN VAY}

Throughout this work we adopt the conventions and notations from [28, Sect. 3] about finite quantum groups which we briefly recall below. Although the results in [28] were stated for non-abelian groups, they also hold for abelian groups. These were proved for instance in [8], [5], [31], [18], [23], [24] by different methods.

Let $\mathbb{k}$ be an algebraically closed field of characteristic zero. We fix a finite group $G$ and a Yetter-Drinfeld module $V \in \mathbb{k}_{\mathbb{k} G}^{\mathbb{V} G \mathcal{D}}$ such that its Nichols algebra $\mathfrak{B}(V)$ is finite-dimensional. Let $\mathfrak{B}(\bar{V})$ be the Nichols algebra of the Yetter-Drinfeld module $\bar{V} \in{\mathbb{\mathbb { k } ^ { G }}}^{\mathrm{G}} \mathcal{Y} \mathcal{D}$ determined by the isomorphism

$$
\mathfrak{B}(\bar{V}) \# \mathbb{k}^{G} \simeq(\mathfrak{B}(V) \# \mathbb{k} G)^{* \mathrm{op}} .
$$

We denote by $\mathcal{D}$ the Drinfeld double of the bosonization $\mathfrak{B}(V) \# \mathbb{k} G$ and by $\mathcal{D}(G)$ the Drinfeld double of $\mathbb{k} G$. Then $\mathfrak{B}(\bar{V}) \# \mathbb{k}^{G}$ and $\mathcal{D}(G)$ are Hopf subalgebras of $\mathcal{D}$. Moreover, $\mathcal{D}$ admits a triangular decomposition, i.e., the multiplication gives a linear isomorphism

$$
\mathfrak{B}(V) \otimes \mathcal{D}(G) \otimes \mathfrak{B}(\bar{V}) \rightarrow \mathcal{D} .
$$

The usual $\mathbb{Z}$-grading on the Nichols algebras induces a $\mathbb{Z}$-grading on $\mathcal{D}$ by setting

$$
\mathcal{D}^{n}=\bigoplus_{n=j-i} \mathfrak{B}^{i}(V) \otimes \mathcal{D}(G) \otimes \mathfrak{B}^{j}(\bar{V}) .
$$

Via the adjoint action in $\mathcal{D}, \bar{V}$ identifies with the dual object of $V$ in the category of $\mathcal{D}(G)$-modules. Moreover, it holds that

$$
\mathfrak{B}^{n}(\bar{V}) \simeq \mathfrak{B}^{n}(V)^{*}
$$

as $\mathcal{D}(G)$-modules for all $n \geq 0$, see the remark below.

The Hopf subalgebra $\mathcal{D}^{\geq 0}$ generated by $\mathfrak{B}(\bar{V})$ and $\mathcal{D}(G)$ satisfies

$$
\mathcal{D}^{\geq 0} \simeq \mathfrak{B}(\bar{V}) \# \mathcal{D}(G) .
$$

Analogously, we will consider the Hopf subalgebra

$$
\mathcal{D}^{\leq 0} \simeq \mathfrak{B}(V) \# \mathcal{D}(G) \text {. }
$$

Remark 1. In [28, Lem. 11(iii) and (iv)] we claim that $\mathfrak{B}(V)$ and $\mathfrak{B}(\bar{V})$ are the Nichols algebras of $V$ and $\bar{V}$ in $\mathcal{D}(G) \mathcal{M}$, and $\mathfrak{B}(\bar{V})$ is isomorphic to $\mathfrak{B}(V)^{* \text { bop }}$ in $\mathcal{D}(G) \mathcal{M}$, the category of $\mathcal{D}(G)$-modules. However, we made a mistake in the proof and these properties do not hold. The correct version of them is the following.

(i) $\mathfrak{B}(\bar{V})$ is isomorphic to the Nichols algebra $\mathfrak{B}\left(\bar{V}, c^{-1}\right)$ corresponding to the dual object of $V$ in ${ }_{\mathcal{D}(G)} \mathcal{M}$ and the inverse braiding of the usual one in $\mathcal{D}(G) \mathcal{M}$.

(ii) There is an isomorphism $\mathfrak{B}(\bar{V}) \simeq \mathfrak{B}(\bar{V}, c)$ of algebras in ${ }_{\mathcal{D}(G)} \mathcal{M}$. Moreover, their defining ideals coincide in the tensor algebra $T(\bar{V})$.

(iii) $\mathfrak{B}^{n}(\bar{V}) \simeq \mathfrak{B}^{n}(V)^{*}$ as $\mathcal{D}(G)$-modules for all $n \geq 0$, that is (1). 
In fact, (i) is a straightforward computation, (ii) follows like [4, Lem. 1.11] and (iii) is a consequence of (ii) and [3, Prop. 3.2.30].

The comultiplication in $\mathcal{D}$ satisfies

$$
\Delta(x) \in x \otimes 1+g_{x} \otimes x+\sum_{i=1}^{n-1}\left(\mathcal{D}^{\leq 0}\right)^{i-n} \otimes\left(\mathcal{D}^{\leq 0}\right)^{-i},
$$

for all $x \in \mathfrak{B}^{n}(V)$ where $g_{x} \in G$, and

$$
\Delta(y) \in y \otimes 1+y_{(-1)} \otimes y_{(0)}+\sum_{i=1}^{n-1}\left(\mathcal{D}^{\geq 0}\right)^{n-i} \otimes\left(\mathcal{D}^{\geq 0}\right)^{i}
$$

for all $y \in \mathfrak{B}^{n}(\bar{V})$ where $y_{(-1)} \otimes y_{(0)}$ is the coaction of $y$ since $\mathfrak{B}^{n}(\bar{V}) \in \mathbb{k}_{\mathbb{k}^{G}}^{\mathbb{Y}^{G}} \mathcal{D}$.

Let $n_{\text {top }}$ be the maximal degree of the Nichols algebra $\mathfrak{B}(V)$. The homogeneous component $\mathfrak{B}^{n_{\text {top }}}(V)$ is one-dimensional and coincides with the space of integrals. We fix a non-zero monomial $x_{\text {top }}=x_{1} \cdots x_{n_{\text {top }}} \in \mathfrak{B}^{n_{\text {top }}}(V)$ with $x_{i} \in V$. The antipode $\mathcal{S}$ applied to this element is

$$
\mathcal{S}\left(x_{\mathrm{top}}\right)=(-1)^{n_{\mathrm{top}}}\left(g_{x_{\mathrm{top}}}^{-1} x_{n_{\mathrm{top}}}\right) \cdots\left(g_{1}^{-1} x_{1}\right)=c x_{\mathrm{top}} g_{x_{\mathrm{top}}}^{-1}
$$

for some non-zero scalar $c$.

The maximal degree of $\mathfrak{B}(\bar{V})$ also is $n_{\text {top }}$. We fix a basis element $y_{\text {top }}$ of $\mathfrak{B}^{n_{\text {top }}}(\bar{V})$.

Let $\beta_{1}$ and $\beta_{2}$ be non-zero right integrals of $\mathfrak{B}(\bar{V}) \# \mathbb{k}^{G}$ and $\mathfrak{B}(V) \# \mathbb{k} G$, respectively. According to our convention, $\mathcal{D}^{*} \simeq\left(\mathfrak{B}(\bar{V}) \# \mathbb{k}^{G}\right) \otimes(\mathfrak{B}(V) \# \mathbb{k} G)$ as algebras. Then $\beta=\beta_{1} \otimes \beta_{2}$ is a non-zero right integral of $\mathcal{D}^{*}$.

The Drinfeld double of a finite-dimensional Hopf algebra is a symmetric algebra, see $[25$, p. 488] and [27], [30]. That is, $\mathcal{D}$ has a non-degenerate bilinear form $(-,-)$ which is associative and symmetric. It is known that $(a, b)=\beta(a b)$ for all $a, b \in \mathcal{D}$. Hence $\left(\mathcal{D}^{n}, \mathcal{D}^{m}\right)=0$, if $n+m \neq 0$.

An important property of a symmetric algebra is that the socle and the head of every indecomposable projective are isomorphic, see for instance $[14,(9.12)]$.

Conventions. In this work we consider finite-dimensional left modules over $\mathcal{D}$. When there is no place for confusion, we will refer to them just as modules. We will use $N, N^{\prime}, N_{1}, \ldots$ to denote them. Projective $\mathcal{D}$-modules are injective (and vice versa) because $\mathcal{D}$ is Frobenius. We will consider the $\mathcal{D}$-modules as $\mathcal{D} \leq 0$-modules or $\mathcal{D}(G)$-modules by restricting the action. We will use $N$ to denote the $\mathcal{D}(G)$ modules.

Let $\mathrm{N}$ be a module and $N$ a $\mathcal{D}(G)$-submodule. We emphasize that the action $\mathfrak{B}(V) \otimes N \rightarrow \mathrm{N}$ is a morphism of $\mathcal{D}(G)$-modules, cf. [28, Equation (31)], and in particular so is $\mathbb{k} x_{\text {top }} \otimes N \rightarrow \mathrm{N}$.

\subsection{Weights}

We fix a representative set $\Lambda$ of isomorphism classes of simple $\mathcal{D}(G)$-modules. We call weights the elements of this set. The counit $\varepsilon$ of $\mathcal{D}(G)$ gives the trivial 


\section{CRISTIAN VAY}

representation. Then, by abuse of notation, we assume that $\varepsilon \in \Lambda$. The dual weight of $\lambda$ will be denoted $\lambda^{*}$.

It is well known that the weights are parametrized by conjugacy classes $\mathcal{O}_{g}$ and irreducible representations $\varrho$ of the centralizer of $g \in G$, see, e.g., [3]. For instance, for $G$ abelian, the weights are one-dimensional and are in bijective correspondence with $G \times \widehat{G}$ where $\widehat{G}$ is the group of characters of $G$. For $G$ non-abelian, the weight $\lambda=M(g, \varrho)$ attached to the pair $(g, \varrho)$ has dimension $\# \mathcal{O}_{g} \cdot \operatorname{dim} \varrho$.

Let $N$ be a $\mathcal{D}(G)$-module and $\lambda \in \Lambda$. We define

$$
[N: \lambda]=\operatorname{dim} \operatorname{Hom}_{\mathcal{D}(G)}(\lambda, N) .
$$

We say that $\lambda$ is a weight of $N$ if $[N: \lambda] \neq 0$. Since $\mathcal{D}(G)$ is a semisimple algebra, $N=\oplus_{\lambda \in \Lambda}[N: \lambda] \cdot \lambda$. In particular, any module over $\mathcal{D}, \mathcal{D} \geq 0$ and $\mathcal{D} \leq 0$ decomposes as $\mathcal{D}(G)$-module into the direct sum of its weights.

Let $K$ be the Grothendieck ring of the category of $\mathcal{D}(G)$-modules. This was described in [33], and anticipated in [26]. We think of $K$ as the free abelian group generated by $\Lambda$. Then the character of $N$ is the element in $K$

$$
\operatorname{ch} N=\sum_{\lambda \in \Lambda}[N: \lambda] \cdot \lambda .
$$

The product in $K$ between $\lambda, \lambda^{\prime} \in \Lambda$ is given by the tensor product

$$
\lambda \cdot \lambda^{\prime}=\operatorname{ch}\left(\lambda \otimes \lambda^{\prime}\right)
$$

Then $\varepsilon$ is the unit of $K$. For $G$ non-abelian $\lambda \otimes \lambda^{\prime}$ could not belong to $\Lambda$ but it decomposes into the direct sum of weights. Recall that $K$ is a commutative ring because $\mathcal{D}(G) \mathcal{M}$ is a braided category.

\subsection{Highest and lowest weights}

A weight is a simple $\mathcal{D}^{\geq 0}$-module if we let $\mathfrak{B}(\bar{V})$ act via the counit. In this case, we call it a highest-weight. Up to isomorphisms, all the simple $\mathcal{D}^{\geq 0}$-modules are highest-weight. A module generated by a highest-weight is called highest-weight module. Notice that the tensor product of highest-weights decomposes into the direct sum of highest-weights since $\Delta(\bar{V}) \subset \bar{V} \otimes 1+\mathbb{k}^{G} \otimes \bar{V}$.

Similarly, we consider weights as $\mathcal{D}^{\leq 0}$-modules and call these lowest-weights. In particular, the space of lowest-weights of a $\mathcal{D}^{\leq 0}$-module $\mathrm{N}$ coincides with its socle

$$
\operatorname{soc}_{\mathcal{D} \leq 0} \mathrm{~N}=\{\mathrm{n} \in \mathrm{N} \mid V \cdot \mathrm{n}=0\} \simeq \oplus_{\lambda \in \Lambda} \operatorname{dim} \operatorname{Hom}_{\mathcal{D} \leq 0}(\lambda, \mathrm{N}) \cdot \lambda .
$$

Since the maximal degree component of a Nichols algebra is one-dimensional,

$$
\lambda_{V}=\operatorname{ch} \mathfrak{B}^{n_{\mathrm{top}}}(V) \quad \text { and } \quad \lambda_{\bar{V}}=\operatorname{ch} \mathfrak{B}^{n_{\mathrm{top}}}(\bar{V})
$$

are one-dimensional weights. These are the unique lowest-weight and highestweight of the regular left modules ${ }_{\mathcal{D} \leq 0} \mathcal{D} \leq 0$ and ${ }_{\mathcal{D} \geq 0} \mathcal{D} \geq 0$. Tensoring by $\lambda_{V}$ induces a bijection between weights because (1) implies

$$
\lambda_{\bar{V}}=\lambda_{V}^{*} \quad \text { and hence } \quad \lambda_{V} \cdot \lambda_{\bar{V}}=\varepsilon .
$$




\subsection{Verma modules}

We fix a weight $\lambda \in \Lambda$. The corresponding Verma module [28, Def. 12] is the induced module

$$
\mathrm{M}(\lambda)=\mathcal{D} \otimes_{\mathcal{D} \geq 0} \lambda
$$

It is a highest-weight module and any highest-weight module of weight $\lambda$ is a quotient of it. By the triangular decomposition, we see that

$$
\mathrm{M}(\lambda) \simeq \mathfrak{B}(V) \otimes \lambda
$$

as $\mathcal{D}(G)$-modules and it is a free $\mathfrak{B}(V)$-module and inherits the $\mathbb{Z}$-grading from $\mathcal{D}$. Verma modules as $\mathcal{D}^{\leq 0}$-modules. Clearly, we have that

$$
\mathrm{M}(\lambda) \simeq \mathcal{D}^{\leq 0} \otimes_{\mathcal{D}(G)} \lambda
$$

and, since the top degree of a Nichols algebra is one-dimensional,

$$
\operatorname{soc}_{\mathcal{D} \leq 0} \mathrm{M}(\lambda)=\mathfrak{B}^{n_{\text {top }}}(V) \otimes \lambda \simeq \lambda_{V} \cdot \lambda .
$$

Lemma 1. As $\mathcal{D}^{\leq 0}$-module, $\mathrm{M}(\lambda)$ is the projective cover of the lowest-weight $\lambda$ and the injective hull of the lowest-weight $\lambda_{V} \cdot \lambda$.

Proof. By (8), $\mathrm{M}(\lambda)$ is an induced module from a semisimple algebra and hence it is projective. Also, it is injective because $\mathcal{D}^{\leq 0}$ is a finite-dimensional Hopf algebra. By $(9), \operatorname{M}(\lambda)$ is indecomposable and the lemma follows.

The next remark is very useful and follows directly from (8).

Remark 2. A morphism $f: \mathrm{M}(\lambda) \rightarrow \mathrm{N}$ of $\mathcal{D}$-modules is injective if and only if $x_{\text {top }} \cdot f(\lambda) \neq 0$.

The dual of a Verma module. For all $\lambda \in \Lambda$ it holds that

$$
\mathrm{M}(\lambda)^{*} \simeq \mathrm{M}\left(\left(\lambda_{V} \cdot \lambda\right)^{*}\right)
$$

In fact, we can see that $\left(\mathfrak{B}^{n_{\mathrm{top}}}(V) \otimes \lambda\right)^{*} \simeq\left(\lambda_{V} \otimes \lambda\right)^{*}$ is a highest-weight of $\mathrm{M}(\lambda)^{*}$ by using the $\mathbb{Z}$-grading. This induces a morphism $f: \mathrm{M}\left(\left(\lambda_{V} \cdot \lambda\right)^{*}\right) \rightarrow \mathrm{M}(\lambda)^{*}$. We apply $f$ to $\operatorname{soc}_{\mathcal{D} \leq 0} \mathrm{M}\left(\left(\lambda_{V} \cdot \lambda\right)^{*}\right)$ and evaluate in the space $1 \otimes \lambda \subset \mathrm{M}(\lambda)$ :

$$
\begin{aligned}
\left\langle x_{\mathrm{top}} \cdot f\left(\left(\lambda_{V} \cdot \lambda\right)^{*}\right), 1 \otimes \lambda\right\rangle & =\left\langle\left(\mathfrak{B}^{n_{\mathrm{top}}}(V) \otimes \lambda\right)^{*}, \mathcal{S}\left(x_{\mathrm{top}}\right) \otimes \lambda\right\rangle \\
& =\left\langle\left(\mathfrak{B}^{n_{\mathrm{top}}}(V) \otimes \lambda\right)^{*}, x_{\mathrm{top}} \otimes g_{x_{\mathrm{top}}}^{-1} \cdot \lambda\right\rangle \neq 0
\end{aligned}
$$

here we use (4) and the fact that the action of $g_{x_{\text {top }}}^{-1}$ is bijective on $\lambda$. Therefore (10) follows from Remark 2 using the finiteness assumption.

For all $0 \leq j \leq n_{\text {top }}$, we immediately deduce from (10) that

$$
\left(\mathfrak{B}^{n_{\text {top }}-j}(V) \otimes \lambda\right)^{*} \simeq \mathfrak{B}^{j}(V) \otimes\left(\lambda_{V} \cdot \lambda\right)^{*}
$$

as $\mathcal{D}(G)$-modules. Using (1), we can rewrite the above isomorphism as

$$
\mathfrak{B}^{n_{\mathrm{top}}-j}(\bar{V}) \otimes \lambda^{*} \simeq\left(\mathfrak{B}^{j}(\bar{V}) \otimes\left(\lambda_{V} \cdot \lambda\right)\right)^{*} .
$$




\section{CRISTIAN VAY}

The co-Verma modules. Let us consider $\lambda$ as a lowest-weight. We set

$$
\mathrm{W}(\lambda)=\mathcal{D} \otimes_{\mathcal{D} \leq 0} \lambda \simeq \mathfrak{B}(\bar{V}) \otimes \lambda
$$

where the isomorphism is of $\mathcal{D}(G)$-modules and it inherits the $\mathbb{Z}$-grading from $\mathcal{D}$.

\subsection{Simple modules}

The Verma modules have simple head and simple socle. This is a well-known fact, see for instance [8], [5], [31], [18] for $G$ abelian, and [23], [24], [28] for general $G$. Therefore the simple $\mathcal{D}$-modules are in bijective correspondence with $\Lambda$.

The co-Verma modules have analogous properties to the Verma modules. In fact, we can see that $W(\lambda)$ have simple head and simple socle as in the proof of [28, Thms. 3 and 4]. These are isomorphic to the socle and the head of $\mathrm{M}\left(\lambda_{\bar{V}} \cdot \lambda\right)$, respectively. Also, they are indecomposable projective as $\mathcal{D}^{\geq 0}$-modules.

We adopt the next conventions.

- $\mathrm{L}(\lambda)$ is the head of $\mathrm{M}(\lambda)$. It is the unique simple highest-weight module of weight $\lambda$. It inherits the $\mathbb{Z}$-grading from $\mathrm{M}(\lambda)[28]$.

- $\mathbf{S}(\lambda)$ is the socle of $\mathrm{M}(\lambda)$. It is the unique simple lowest-weight module of weight $\lambda_{V} \cdot \lambda$. The lowest-weight of $\mathrm{S}(\lambda)$ is realized by $\mathfrak{B}^{n_{\text {top }}}(V) \otimes \lambda$.

- $\bar{\lambda}$ denotes the lowest-weight of $\mathrm{L}(\lambda)$. Then the assignment $\lambda \mapsto \bar{\lambda}$ is a bijection in $\Lambda$.

- We think of M, W, L and S (and also P and Ind, see below) as maps from $K$ to $\mathcal{D} \mathcal{M}$ which transform sums of weights into direct sums of modules. We will use this fact to abbreviate the notation. For instance, we will write $\mathrm{M}(\lambda \cdot \mu)$ instead of $\oplus_{i} n_{i} \cdot \mathrm{M}\left(\lambda_{i}\right)$ if $\lambda \cdot \mu=\sum_{i} n_{i} \lambda_{i}$.

Then

$$
\mathrm{L}(\lambda) \simeq \mathrm{S}\left(\lambda_{\bar{V}} \cdot \bar{\lambda}\right) \text { and } \mathrm{L}(\lambda)^{*} \simeq \mathrm{L}\left(\bar{\lambda}^{*}\right)
$$

the first isomorphism follows by the characterization of the simple modules and the second one follows by [28, Thm. 5].

\subsection{Projective modules}

The next lemma is inspired by [25, Lem. 2.4].

Lemma 2. Let $\mathrm{P}$ be projective. Then

$$
\operatorname{soc}_{\mathcal{D} \leq 0} \mathrm{P} \simeq \bigoplus_{\lambda \in \Lambda} \operatorname{dim} \operatorname{Hom}_{\mathcal{D} \leq 0}(\lambda, \mathrm{P}) \cdot \lambda,
$$

if and only if, as $\mathcal{D}^{\leq 0}$-modules,

$$
\mathrm{P} \simeq \bigoplus_{\lambda \in \Lambda} \operatorname{dim} \operatorname{Hom}_{\mathcal{D} \leq 0}(\lambda, \mathrm{P}) \cdot \mathrm{M}\left(\lambda_{\bar{V}} \cdot \lambda\right)
$$

Proof. Since $\mathcal{D}$ is free over $\mathcal{D} \leq 0, \mathrm{P}$ is $\mathcal{D}^{\leq 0}$-projective. Hence $\mathrm{P}$ decomposes into the direct sum of indecomposable projective $\mathcal{D}^{\leq 0}$-modules, which are precisely the Verma modules by Lemma 1. Then, the lemma follows using (9). 


\section{ON PROJECTIVE MODULES OVER FINITE QUANTUM GROUPS}

Definition 1. Given a weight $\lambda, P(\lambda)$ denotes the projective cover of the simple highest-weight module $\mathrm{L}(\lambda)$.

Lemma 3. Let $\lambda$ be a weight. Then

(i) $\mathrm{P}(\lambda)$ is the injective hull of $\mathrm{L}(\lambda)$.

(ii) $\mathrm{P}(\lambda)$ is the projective cover of $\mathrm{M}(\lambda)$.

(iii) $\mathrm{P}(\lambda)$ is the injective hull of $\mathrm{M}\left(\lambda_{\bar{V}} \cdot \bar{\lambda}\right)$.

(iv) $\mathrm{P}(\lambda)$ is the projective cover of $\mathrm{W}(\bar{\lambda})$.

(v) $\mathrm{P}(\lambda)$ is the injective hull of $\mathrm{W}\left(\lambda_{V} \cdot \lambda\right)$.

(vi) $\mathrm{P}(\lambda)^{*} \simeq \mathrm{P}\left(\bar{\lambda}^{*}\right)$.

Proof. (i) holds because $\mathcal{D}$ is a symmetric algebra.

(ii), (iii) As the Verma modules have simple socle and simple head, we can deduce that $\mathrm{P}(\lambda)$ is the projective cover of $\mathrm{M}(\lambda)$ and the injective hull of $\mathrm{M}\left(\lambda_{\bar{V}} \cdot \bar{\lambda}\right)$, see for instance $[14,(6.25)]$.

We show (iv) and (v) in a similar way, cf. (13).

(vi) is a consequence of (i) and (14) because $\mathrm{P}(\lambda)^{*}$ is the injective hull of $\mathrm{L}(\lambda)^{*}$.

By the above lemma we have the next commutative diagrams of injective and surjective morphisms:
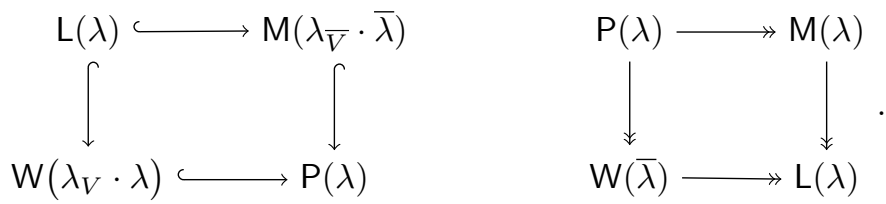

We deduce from the morphisms in the first rows that

$$
\bar{\lambda} \text { and } \lambda_{V} \cdot \lambda \text { are weights of } \operatorname{soc}_{\mathcal{D} \leq 0} \mathrm{P}(\lambda)
$$

The numbers of occurrences of $\mathrm{L}(\lambda)$ as composition factor of $\mathrm{N}$ will be denoted by $[N: L(\lambda)]$. It is known that $[N: L(\lambda)]=\operatorname{dim} \operatorname{Hom}_{\mathcal{D}}(P(\lambda), N)=\operatorname{dim} \operatorname{Hom}_{\mathcal{D}}(N, P(\lambda))$, the last equality is thanks to Lemma 3(i).

\subsection{Induced modules}

Definition 2. Given a weight $\lambda$, Ind $(\lambda)$ denotes the induced module $\mathcal{D} \otimes_{\mathcal{D}(G)} \lambda$.

We can consider Ind $(\lambda)$ as a submodule of the left regular module ${ }_{\mathcal{D}} \mathcal{D}$ since $\mathcal{D}(G)$ is a semisimple subalgebra. Moreover, it is a direct summand and hence projective. Then

$$
\operatorname{lnd}(\lambda)=\oplus_{\mu \in \Lambda}[\mathrm{L}(\mu): \lambda] \cdot \mathrm{P}(\mu)
$$

In fact, if $\lambda$ is a weight of $\mathrm{L}(\mu)$, then there is a non-trivial epimorphism $f: \operatorname{lnd}(\lambda) \rightarrow$ $\mathrm{L}(\mu)$ just by the definition of induced module. Thus $f$ factors through $\mathrm{P}(\mu)$ and we deduce (17) because of the Frobenius reciprocity $\operatorname{Hom}_{\mathcal{D}}(\operatorname{lnd}(\lambda), \mathrm{L}(\mu)) \simeq$ $\operatorname{Hom}_{\mathcal{D}(G)}(\lambda, \mathrm{L}(\mu))$. 


\section{CRISTIAN VAY}

The triangular decomposition of $\mathcal{D}$ gives linear isomorphisms

$$
\operatorname{lnd}(\lambda) \simeq \mathfrak{B}(\bar{V}) \otimes \mathfrak{B}(V) \otimes \lambda \simeq \mathfrak{B}(V) \otimes \mathfrak{B}(\bar{V}) \otimes \lambda
$$

and $\operatorname{lnd}(\lambda)$ inherits the $\mathbb{Z}$-grading of $\mathcal{D}$. Therefore we see that

$$
\operatorname{soc}_{\mathcal{D} \leq 0} \operatorname{lnd}(\lambda) \simeq \lambda_{V} \otimes \mathfrak{B}(\bar{V}) \otimes \lambda .
$$

Hence, by Lemma 2, we have the next isomorphism of $\mathcal{D} \leq 0$-modules:

$$
\operatorname{lnd}(\lambda) \simeq \mathrm{M}(\operatorname{ch} \mathfrak{B}(\bar{V}) \cdot \lambda) .
$$

Lemma 4. Let $\lambda, \mu$ be weights and

$$
f: \operatorname{lnd}(\lambda \cdot \mu) \rightarrow \mathrm{W}(\lambda) \otimes \mathrm{M}(\mu)
$$

the morphism induced by $\lambda \otimes \mu \stackrel{\sim}{\rightarrow}(1 \otimes \lambda) \otimes(1 \otimes \mu)$. Then $f$ is an isomorphism.

Proof. We will see that $f$ is injective using Remark 2 and hence $f$ is an isomorphism because the modules have the same dimension.

Let $z \in \operatorname{soc}_{\mathcal{D} \leq 0} \operatorname{Ind}(\lambda \cdot \mu)$. By (19), $z=x_{\text {top }} \sum_{i} y_{i}\left(h_{i} \otimes k_{i}\right)$ where $y_{i} \in \mathfrak{B}(\bar{V})$ and $\left(h_{i} \otimes k_{i}\right) \in \lambda \otimes \mu$. Then

$$
f(z)=x_{\mathrm{top}} \sum\left(y_{i} h_{i}\right) \otimes k_{i} \in \sum g_{x_{\mathrm{top}}}\left(y_{i} h_{i}\right) \otimes\left(x_{\mathrm{top}} k_{i}\right)+\mathrm{W}(\lambda) \otimes\left(\bigoplus_{i=0}^{n_{\mathrm{top}}-1} \mathrm{M}^{-i}(\mu)\right),
$$

using that $\mu$ is a highest-weight, (2) and (3). Then $f$ is injective in $\operatorname{soc}_{\mathcal{D} \leq 0} \operatorname{lnd}(\lambda \cdot \mu)$ because $g_{x_{\text {top }}} \otimes g_{x_{\text {top }}} \otimes x_{\text {top }}: \mathfrak{B}(\bar{V}) \otimes \lambda \otimes \mu \rightarrow \mathfrak{B}(\bar{V}) \otimes \lambda \otimes \mathfrak{B}^{n_{\text {top }}}(V) \mu$ is injective.

\subsection{Tensor identity}

The next lemma is well known, see for instance [16, Prop. 1.7] and the remark after it.

Lemma 5. Let $A$ be a Hopf algebra and $B$ a Hopf subalgebra of $A$. Let $\mathrm{N}$ be an $A$-module, $U$ a $B$-module and $U \otimes \mathrm{N} \rightarrow\left(A \otimes_{B} U\right) \otimes \mathrm{N}$ the inclusion of $B$-modules given by $u \otimes \mathrm{n} \mapsto 1 \otimes u \otimes \mathrm{n}$. Then the induced morphism

$$
f: A \otimes_{B}(U \otimes \mathrm{N}) \rightarrow\left(A \otimes_{B} U\right) \otimes \mathrm{N}
$$

is an isomorphism of A-modules.

We can apply the above to $A=\mathcal{D}$ and $B=\mathcal{D}^{\geq 0}$.

Lemma 6. Let $\mathrm{M}(\lambda)$ be a Verma module and $\mu$ a highest-weight of a module $\mathrm{N}$. Then $\mathrm{M}(\lambda \cdot \mu)$ is a submodule of $\mathrm{M}(\lambda) \otimes \mathrm{N}$.

Proof. By assumption we have an inclusion $\lambda \otimes \mu \rightarrow \lambda \otimes \mathrm{N}$ of $\mathcal{D}^{\geq 0}$-modules and the functor $\mathcal{D} \otimes_{\mathcal{D} \geq 0}(-)$ is exact because $\mathcal{D}$ is a free $\mathcal{D}^{\geq 0}$-module. Then

$$
\mathrm{M}(\lambda \cdot \mu)=\mathcal{D} \otimes_{\mathcal{D} \geq 0}(\lambda \otimes \mu) \rightarrow \mathcal{D} \otimes_{\mathcal{D} \geq 0}(\lambda \otimes \mathrm{N})
$$

is an inclusion of $\mathcal{D}$-modules and the lemma follows by Lemma 5 .

Lemma 7. Let $\mathrm{P}$ be the injective hull of $\mathrm{M}\left(\lambda_{\bar{V}} \cdot \bar{\lambda} \cdot \mu\right)$ for some weights $\lambda$ and $\mu$. Then $\mathrm{P}$ is a direct summand of $\mathrm{P}(\lambda) \otimes \mathrm{L}(\mu)$.

Proof. By Lemma 3 iii, $\mathrm{P}(\lambda)$ is the injective hull of $\mathrm{M}\left(\lambda_{\bar{V}} \cdot \bar{\lambda}\right)$. By Lemma 6, $\mathrm{M}\left(\lambda_{\bar{V}} \cdot \bar{\lambda} \cdot \mu\right)$ is a submodule of $\mathrm{P}(\lambda) \otimes \mathbf{L}(\mu)$. Since $\mathrm{P}(\lambda) \otimes \mathbf{L}(\mu)$ is injective, the lemma follows. 


\section{ON PROJECTIVE MODULES OVER FINITE QUANTUM GROUPS}

\section{Graded modules}

We start by summarizing some notions about graded modules.

Let $\mathrm{N}=\bigoplus_{i \in \mathbb{Z}} \mathrm{N}(i)$ be a $\mathbb{Z}$-graded $\mathcal{D}$-module, that is $\mathcal{D}^{n} \cdot \mathrm{N}(i) \subseteq \mathrm{N}(n+i)$ for all $n, i \in \mathbb{Z}$. A morphism $f: \mathrm{N} \rightarrow \mathrm{N}^{\prime}$ between $\mathbb{Z}$-graded $\mathcal{D}$-modules has degree $\ell$ if $f(\mathrm{~N}(i)) \subseteq \mathrm{N}^{\prime}(i+\ell)$. The space of such morphisms is denoted by $\operatorname{Hom}_{\mathcal{D}}^{\bullet}\left(\mathrm{N}, \mathrm{N}^{\prime}\right)_{\ell}$. Thus, the morphisms in the category of $\mathbb{Z}$-graded $\mathcal{D}$-modules are the 0 -degree ones. The $\ell$-shift $T_{\ell}$ is an endofunctor in the category of $\mathbb{Z}$-graded $\mathcal{D}$-modules such that $\mathrm{N}[\ell]=T_{\ell}(\mathrm{N})$ is $\mathrm{N}$ as $\mathcal{D}$-module with homogeneous components $\mathrm{N}[\ell](i)=\mathrm{N}(i-\ell)$ for all $i \in \mathbb{Z}$. These functors satisfy $T_{\ell} \circ T_{\ell^{\prime}}=T_{\ell+\ell^{\prime}}$ for all $\ell, \ell^{\prime} \in \mathbb{Z}$.

The Hom-spaces satisfy $\operatorname{Hom}_{\mathcal{D}}^{\bullet}\left(\mathrm{N}[\ell], \mathrm{N}^{\prime}\right)_{0}=\operatorname{Hom}_{\mathcal{D}}\left(\mathrm{N}, \mathrm{N}^{\prime}\right)_{\ell}=\operatorname{Hom}_{\mathcal{D}}\left(\mathrm{N}, \mathrm{N}^{\prime}[-\ell]\right)_{0}$ and

$$
\operatorname{Hom}_{\mathcal{D}}\left(\mathrm{N}, \mathrm{N}^{\prime}\right)=\bigoplus_{\ell \in \mathbb{Z}} \operatorname{Hom}_{\mathcal{D}}^{\bullet}\left(\mathrm{N}, \mathrm{N}^{\prime}\right)_{\ell}
$$

Given a Laurent polynomial $p=\sum_{i \in \mathbb{Z}} a_{i} t^{i} \in \mathbb{Z}_{\geq 0}\left[t, t^{-1}\right]$, we set

$$
p \cdot \mathrm{N}=\bigoplus_{i \in \mathbb{Z}} a_{i} \cdot T_{i}(\mathrm{~N})
$$

This induces an action of $\mathbb{Z}\left[t, t^{-1}\right]$ over the Grothendieck ring $R^{\bullet}$ of the category of $\mathbb{Z}$-graded $\mathcal{D}$-modules.

The modules $\mathrm{L}(\lambda)[i]$, with $\lambda \in \Lambda$ and $i \in \mathbb{Z}$, form a complete set of nonisomorphic simple $\mathbb{Z}$-graded $\mathcal{D}$-modules.

Since each $\mathrm{N}(i)$ is a $\mathcal{D}(G)$-module, we define the graded character of $\mathrm{N}$ by setting

$$
\operatorname{ch}^{\bullet} \mathrm{N}=\sum_{i \in \mathbb{Z}} \operatorname{ch} \mathrm{N}(i) t^{i} \in K\left[t, t^{-1}\right]
$$

Clearly, there exist unique $p_{\mathrm{N}, \lambda} \in \mathbb{Z}\left[t, t^{-1}\right], \lambda \in \Lambda$, such that

$$
\operatorname{ch}^{\bullet} \mathrm{N}=\sum_{\lambda \in \Lambda} p_{\mathrm{N}, \lambda} \cdot \lambda
$$

The tensor product between $\mathbb{Z}$-graded $\mathcal{D}$-modules is $\mathbb{Z}$-graded with the natural grading for a tensor product and it holds that

$$
\operatorname{ch}^{\bullet}\left(\mathrm{N} \otimes \mathrm{N}^{\prime}\right)=\operatorname{ch}^{\bullet} \mathrm{N} \cdot \mathrm{ch}^{\bullet} \mathrm{N}^{\prime}
$$

The dual of $\mathrm{N}$ also is a $\mathbb{Z}$-graded $\mathcal{D}$-module by setting

$$
\mathrm{N}^{*}(j)=(\mathrm{N}(-j))^{*}
$$

This is compatible with the grading of $\mathcal{D}$ because the antipode is an homogeneous morphism.

Therefore $\mathrm{ch}^{\bullet}: R^{\bullet} \rightarrow K\left[t, t^{-1}\right]$ is a ring homomorphism and $\mathrm{ch}^{\bullet} \mathrm{N}^{*}=\overline{\mathrm{ch}^{\bullet} \mathrm{N}}$, where $\overline{p\left(t, t^{-1}\right)}=p\left(t^{-1}, t\right)$ for any $p \in K\left[t, t^{-1}\right]$. 


\section{CRISTIAN VAY}

\subsection{Graded projectives}

Thanks to [17, Cor. 3.4] every projective module admits a $\mathbb{Z}$-grading. Moreover, up to a shift, every indecomposable projective module admits only one $\mathbb{Z}$-grading [17, Thm. 4.1].

For each $\lambda \in \Lambda$ we fix a $\mathbb{Z}$-grading $\mathrm{P}(\lambda)=\bigoplus_{n \in \mathbb{Z}} \mathrm{P}(\lambda)(n)$ such that there is a homogeneous weight $S$ of degree 0 generating $\mathrm{P}(\lambda)$ with $\operatorname{ch} S=\lambda$. Thus, we have a commutative diagram of $\mathbb{Z}$-graded $\mathcal{D}$-module epimorphisms and a section

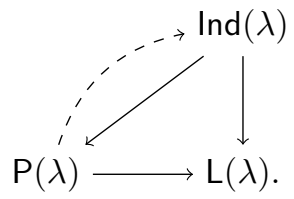

Also, the diagram on the right hand of (15) is of $\mathbb{Z}$-graded $\mathcal{D}$-modules.

Given $\lambda \in \Lambda$, we denote $l_{\lambda}$ the minimal degree of $\mathrm{L}(\lambda)$. Thus, (14) implies that

$$
(\mathrm{L}(\lambda)[i])^{*} \simeq \mathrm{L}\left(\bar{\lambda}^{*}\right)\left[-i-l_{\lambda}\right] \quad \text { and } \quad l_{\lambda}=l_{\bar{\lambda}^{*}}
$$

Lemma 8. Let $\lambda$ be a weight and $i \in \mathbb{Z}$. In the category of $\mathbb{Z}$-graded $\mathcal{D}$-modules it holds that

(i) $\mathrm{P}(\lambda)[i]$ is the projective cover and the injective hull of $\mathrm{L}(\lambda)[i]$.

(ii) $\mathrm{P}(\lambda)[i]$ is the projective cover of $\mathrm{M}(\lambda)[i]$.

(iii) $\mathrm{P}(\lambda)[i]$ is the injective hull of $\mathrm{M}\left(\lambda_{\bar{V}} \cdot \bar{\lambda}\right)\left[i+l_{\lambda}+n_{\mathrm{top}}\right]$.

(iv) $\mathrm{P}(\lambda)[i]$ is the projective cover of $\mathrm{W}(\bar{\lambda})\left[i+l_{\lambda}\right]$.

(v) $\mathrm{P}(\lambda)[i]$ is the injective hull of $\mathrm{W}\left(\lambda_{V} \cdot \lambda\right)\left[i-n_{\mathrm{top}}\right]$.

(vi) $(\mathrm{P}(\lambda)[i])^{*} \simeq \mathrm{P}\left(\bar{\lambda}^{*}\right)\left[-i-l_{\lambda}\right]$.

Proof. (i) The first part is clear. Let $e \in \mathcal{D}^{0}$ be a primitive idempotent such that $\mathrm{P}(\lambda) \simeq \mathcal{D} e[17$, Prop. 5.8(iii)], and $\mathrm{S}$ be the socle of $\mathcal{D} e$ which is homogeneous by [17, Prop. 3.5(ii)]. Since the symmetric bilinear form of $\mathcal{D}$ satisfies $(1, e \mathrm{~S}(0))=$ $(1, e \mathrm{~S})$, recall Section 2, we can argue as in the proof of $[14,(9.12)]$ to show that $e \mathrm{~S}(0) \neq 0$. Hence $\mathrm{P}(\lambda)[i]$ is the injective hull of $\mathrm{L}(\lambda)[i]$.

(vi) follows from Lemma $3(\mathrm{i})$ and (26).

The remaining items are deduced from (i) and Lemma 3(iii).

Let $\mathrm{P}$ be a projective module with a fixed $\mathbb{Z}$-grading. Therefore there exist unique polynomials $p_{\mathrm{P}, \mathrm{P}(\lambda)} \in \mathbb{Z}_{\geq 0}\left[t, t^{-1}\right]$ such that

$$
\mathrm{P} \simeq \bigoplus_{\lambda \in \Lambda} p_{\mathrm{P}, \mathrm{P}(\lambda)} \cdot \mathrm{P}(\lambda) \text { if and only if } \operatorname{ch}^{\bullet} \mathrm{P}=\sum_{\lambda \in \Lambda} p_{\mathrm{P}, \mathrm{P}(\lambda)} \operatorname{ch}^{\bullet} \mathrm{P}(\lambda)
$$

by $\left[17\right.$, Prop. 5.8 (iii)]. If $[\mathrm{P}: \mathrm{P}(\lambda)]=p_{\mathrm{P}, \mathrm{P}(\lambda)}(1)$, then

$$
\mathrm{P} \simeq \bigoplus_{\lambda \in \Lambda}[\mathrm{P}: \mathrm{P}(\lambda)] \cdot \mathrm{P}(\lambda)
$$

as ungraded modules. 


\section{ON PROJECTIVE MODULES OVER FINITE QUANTUM GROUPS}

Let us consider $\mathrm{P}$ as a $\mathcal{D}^{\leq 0}$-module. Since $\mathcal{D}^{\leq 0}$ is a graded subalgebra of $\mathcal{D}$, $P$ also is a projective $\mathbb{Z}$-graded $\mathcal{D}^{\leq 0}$-module. Hence, by [17, Prop. 5.8(iii)] and Lemma 1 , there exist unique polynomials $p_{\mathrm{P}, \mathrm{M}(\lambda)} \in \mathbb{Z}_{\geq 0}\left[t, t^{-1}\right]$ such that

$$
\mathrm{P} \simeq \bigoplus_{\lambda \in \Lambda} p_{\mathrm{P}, \mathrm{M}(\lambda)} \cdot \mathrm{M}(\lambda) \quad \text { as } \mathcal{D}^{\leq 0} \text {-modules }
$$

if and only if

$$
\operatorname{ch}^{\bullet} \mathrm{P}=\sum_{\lambda \in \Lambda} p_{\mathrm{P}, \mathrm{M}(\lambda)} \operatorname{ch}^{\bullet} \mathrm{M}(\lambda)
$$

Moreover, for each $\lambda \in \Lambda$ and $i \in \mathbb{Z}$, we assume that the lowest-weight $\lambda_{V} \cdot \lambda$ is concentrated in degree 0 and set

$$
a_{\mathrm{P}, \mathrm{M}(\lambda), i}=\operatorname{dim} \operatorname{Hom}_{\mathcal{D} \leq 0}^{\bullet}\left(\lambda_{V} \cdot \lambda, \mathrm{P}\right)_{i-n_{\mathrm{top}}} .
$$

Therefore $\operatorname{dim} \operatorname{Hom}_{\mathcal{D} \leq 0}\left(\lambda_{V} \cdot \lambda, \mathrm{P}\right)=p_{\mathrm{P}, \mathrm{M}(\lambda)}(1)$ and

$$
p_{\mathrm{P}, \mathrm{M}(\lambda)}=\sum_{i} a_{\mathrm{P}, \mathrm{M}(\lambda), i} t^{i} \in \mathbb{Z}\left[t, t^{-1}\right]
$$

by Lemma 2 .

In particular, we have that $p_{\operatorname{Ind}(\mu), \mathrm{P}(\lambda)}=\overline{p_{\mathrm{L}(\lambda), \mu}}$ and $p_{\operatorname{Ind}(\mu), \mathrm{M}(\lambda)}=p_{\mathfrak{B}(\bar{V}) \otimes \mu, \lambda}$ for all $\lambda, \mu \in \Lambda$. Thus, we obtain graded versions of (17) and (20), that is

$$
\operatorname{lnd}(\mu) \simeq \oplus_{\lambda \in \Lambda} \overline{p_{\mathrm{L}(\lambda), \mu}} \cdot \mathrm{P}(\lambda) \simeq \mathrm{M}\left(\operatorname{ch}^{\bullet} \mathfrak{B}(\bar{V}) \cdot \mu\right)
$$

as $\mathbb{Z}$-graded $\mathcal{D}$-modules and $\mathbb{Z}$-graded $\mathcal{D} \leq 0$-modules, respectively.

Remark 3. Let $R_{\text {proj }}^{\bullet}$ be the Grothendieck ring of the subcategory of projective modules. Clearly, $\left\{\mathrm{ch}^{\bullet} \mathrm{P}(\lambda) \mid \lambda \in \Lambda\right\}$ is a $\mathbb{Z}\left[t, t^{-1}\right]$-bases of $R_{\text {proj }}^{\bullet}$. Moreover, the sets $\left\{\operatorname{ch}^{\bullet} \mathrm{M}(\lambda) \mid \lambda \in \Lambda\right\}$ and $\left\{\mathrm{ch}^{\bullet} \mathrm{W}(\lambda) \mid \lambda \in \Lambda\right\}$ so are by (29) and (38).

\subsection{The simple modules are identified by their graded characters}

Let $\mathbf{N}=\bigoplus_{i \in \mathbb{Z}} \mathbf{N}(i)$ be a $\mathbb{Z}$-graded $\mathcal{D}$-module. For each $\lambda \in \Lambda$ and $i \in \mathbb{Z}$, we define

$$
a_{\mathrm{N}, \mathrm{L}(\lambda), i}=\operatorname{dim} \operatorname{Hom}_{\mathcal{D}}^{\bullet}(\mathrm{P}(\lambda)[i], \mathrm{N})_{0}=\operatorname{dim} \operatorname{Hom}_{\mathcal{D}}^{\bullet}(\mathrm{N}, \mathrm{P}(\lambda)[i])_{0} ;
$$

these dimensions are equal by Lemma $8(\mathrm{i})$. It is the number of composition factors of $\mathrm{N}$ isomorphic to $\mathrm{L}(\lambda)[i]$. We also define the Laurent polynomial

$$
p_{\mathrm{N}, \mathrm{L}(\lambda)}=\sum_{i} a_{\mathrm{N}, \mathrm{L}(\lambda), i} t^{i} \in \mathbb{Z}\left[t, t^{-1}\right] .
$$

By the next theorem the ring morphism $\mathrm{ch}^{\bullet}: R^{\bullet} \rightarrow K\left[t, t^{-1}\right]$ is injective.

Theorem 9. The set $\left\{\operatorname{ch}^{\bullet} \mathrm{L}(\lambda) \mid \lambda \in \Lambda\right\}$ is a $\mathbb{Z}\left[t, t^{-1}\right]$-basis of $R^{\bullet}$. More explicitly, if $\mathrm{N}=\bigoplus_{i \in \mathbb{Z}} \mathrm{N}(i)$ is a $\mathbb{Z}$-graded $\mathcal{D}$-module, then the Laurent polynomials $p_{\mathrm{N}, \mathrm{L}(\lambda)}$ are the unique ones such that

$$
\operatorname{ch}^{\bullet} \mathrm{N}=\sum_{\lambda \in \Lambda} p_{\mathrm{N}, \mathrm{L}(\lambda)} \operatorname{ch}^{\bullet} \mathrm{L}(\lambda) .
$$

Therefore $[\mathrm{N}: \mathrm{L}(\lambda)]=p_{\mathrm{N}, \mathrm{L}(\lambda)}(1)$. 


\section{CRISTIAN VAY}

Proof. Suppose that $0=\sum_{\lambda \in \Lambda} p_{\lambda} \mathrm{ch}^{\bullet} \mathrm{L}(\lambda)$ with $p_{\lambda} \in \mathbb{Z}\left[t, t^{-1}\right]$. Multiplying by a suitable $t^{j}$ with $j<0$, we can assume that $p_{\lambda} \in \mathbb{Z}\left[t^{-1}\right]$ for all $\lambda$. Then, we deduce that $p_{\lambda}=0$ for all $\lambda$ since $\operatorname{ch}^{\bullet} \mathrm{L}(\lambda)=\lambda+\sum_{j<0} \operatorname{ch}(\mathrm{L}(\lambda)(j)) t^{j}$, and the uniqueness follows.

Let $\mathrm{L}$ be a simple submodule of $N$. As $\mathrm{L}$ is graded and a highest-weight module, there is $i \in \mathbb{Z}$ such that $\mathrm{L}(j) \subseteq \mathrm{N}(i+j)$ and the quotient $\mathrm{N} / \mathrm{L}$ is a $\mathbb{Z}$-graded $\mathcal{D}$ module. Then $\mathrm{ch}^{\bullet} \mathrm{N}=t^{i} \mathrm{ch}^{\bullet} \mathrm{L}+\mathrm{ch}^{\bullet} \mathrm{N} / \mathrm{L}$ and the theorem follows by induction on the length of $\mathbf{N}$; we use that $\mathrm{P}(\lambda)[i]$ is a $\mathbb{Z}$-graded projective module and then $\operatorname{Hom}_{\mathcal{D}}(\mathrm{P}(\lambda)[i],-)_{0}$ is an exact functor.

The equality $[\mathrm{N}: \mathrm{L}(\lambda)]=p_{\mathrm{N}, \mathrm{L}(\lambda)}(1)$ is clear.

\subsection{Graded character identities}

Irving has defined a class of highest weight categories for which the BGG Reciprocity holds [21]. These categories have a duality functor $\delta$ which is trivial on simple modules. Using the order on the weights, he shows that the characters of a Verma module $M$ and $\delta M$ are equal.

Although we do not have either such a duality or an order on the weights, we can prove the following identities among the graded characters of Verma modules, their duals and the modules $\mathrm{W}(\lambda)=\mathcal{D} \otimes_{\mathcal{D} \leq 0} \lambda$, recall (13). Such identities are key in the proof of the BGG Reciprocity.

Theorem 10. Let $\lambda$ be a weight. Then

$$
t^{n_{\text {top }}} \operatorname{ch}^{\bullet} \mathrm{W}\left(\lambda_{V} \cdot \lambda\right)^{*} \underset{(\mathrm{i})}{=} \operatorname{ch}^{\bullet} \mathrm{W}\left(\lambda^{*}\right) \underset{(\mathrm{ii})}{=} \operatorname{ch}^{\bullet} \mathrm{M}(\lambda)^{*} \underset{(\mathrm{iii})}{=} t^{n_{\text {top }}} \operatorname{ch}^{\bullet} \mathrm{M}\left(\left(\lambda_{V} \cdot \lambda\right)^{*}\right) .
$$

Proof. We have that $\left(\mathfrak{B}^{j}(V) \otimes \lambda\right)^{*} \simeq \mathfrak{B}^{j}(V)^{*} \otimes \lambda^{*} \simeq \mathfrak{B}^{j}(\bar{V}) \otimes \lambda^{*}$ by (1) and hence $\operatorname{ch}^{\bullet} \mathrm{W}\left(\lambda^{*}\right)=\operatorname{ch}^{\bullet} \mathrm{M}(\lambda)^{*}$, it is (ii).

On the other hand, (11) and (12) imply (i) and (iii), respectively:

$$
\operatorname{ch}^{\bullet} \mathrm{M}(\lambda)^{*}=t^{n_{\mathrm{top}}} \mathrm{ch}^{\bullet} \mathrm{M}\left(\left(\lambda_{V} \cdot \lambda\right)^{*}\right) \quad \text { and } \quad t^{n_{\mathrm{top}}} \mathrm{ch}^{\bullet} \mathrm{W}\left(\lambda_{V} \cdot \lambda\right)^{*}=\operatorname{ch}^{\bullet} \mathrm{W}\left(\lambda^{*}\right) .
$$

We compare the graded composition factors of a Verma module and its dual using that $\left\{\operatorname{ch}^{\bullet} \mathrm{L}(\lambda) \mid \lambda \in \Lambda\right\}$ is a $\mathbb{Z}\left[t, t^{-1}\right]$-basis. Recall that $\mathrm{M}(\mu)^{*} \simeq \mathrm{M}\left(\left(\lambda_{V} \cdot \mu\right)^{*}\right)$ by $(10)$.

Corollary 11. Let $\lambda$ and $\mu$ be weights. Then

$$
t^{l_{\mu}+n_{\mathrm{top}}} p_{\mathrm{M}(\lambda), \mathrm{L}(\mu)}=\overline{p_{\mathrm{M}\left(\left(\lambda_{V} \cdot \lambda\right)^{*}\right), \mathrm{L}\left(\bar{\mu}^{*}\right)}} .
$$

In particular, $[\mathrm{M}(\lambda): \mathrm{L}(\mu)]=\left[\mathrm{M}\left(\left(\lambda_{V} \cdot \lambda\right)^{*}\right): \mathrm{L}\left(\bar{\mu}^{*}\right)\right]$ by evaluating the above polynomials at $t=t^{-1}=1$.

Proof. We have the linear isomorphisms

$$
\begin{aligned}
\operatorname{Hom}_{\mathcal{D}}^{\bullet}(\mathrm{P}(\mu)[i], \mathrm{M}(\lambda))_{0} & \simeq \operatorname{Hom}_{\mathcal{D}}^{\bullet}\left(\mathrm{M}(\lambda)^{*},(\mathrm{P}(\mu)[i])^{*}\right)_{0} \\
& \simeq \operatorname{Hom}_{\mathcal{D}}^{\bullet}\left(\mathrm{M}(\lambda)^{*}, \mathrm{P}\left(\bar{\mu}^{*}\right)\left[-l_{\mu}-i\right]\right)_{0} .
\end{aligned}
$$

Then $a_{\mathrm{M}(\lambda), \mathrm{L}(\mu), i}=a_{\mathrm{M}(\lambda)^{*}, \mathrm{~L}\left(\bar{\mu}^{*}\right),-l_{\mu}-i}$ and hence $p_{\mathrm{M}(\lambda), \mathrm{L}(\mu)}=t^{-l_{\mu}} \overline{p_{\mathrm{M}(\lambda)^{*}, \mathrm{~L}\left(\bar{\mu}^{*}\right)}}$. Thus, the corollary follows from Theorem 10 . 
We do not know yet whether the projective modules are filtered by Verma modules. Instead, we know that they decompose into the direct sum of Verma modules as $\mathbb{Z}$-graded $\mathcal{D}^{\leq 0}$-modules (29). Such decomposition is related to the graded composition factors of the Verma modules, as we see below in a graded version of the BGG Reciprocity.

Corollary 12. Let $\lambda$ and $\mu$ be weights. Then

$$
p_{\mathrm{P}(\mu), \mathrm{M}(\lambda)}=\overline{p_{\mathrm{M}(\lambda), \mathrm{L}(\mu)}} .
$$

Proof. We have the linear isomorphisms

$$
\begin{aligned}
\operatorname{Hom}_{\mathcal{D} \leq 0}^{\bullet}\left(\lambda_{V} \cdot \lambda, \mathrm{P}(\mu)\right)_{i-n_{\text {top }}} & \simeq \operatorname{Hom}_{\mathcal{D}}\left(\mathrm{W}\left(\lambda_{V} \cdot \lambda\right), \mathrm{P}(\mu)\right)_{i-n_{\text {top }}} \\
& \simeq \operatorname{Hom}_{\mathcal{D}}\left(\mathrm{P}(\mu)^{*},\left(\mathrm{~W}\left(\lambda_{V} \cdot \lambda\right)\left[i-n_{\text {top }}\right]\right)^{*}\right)_{0} \\
& \simeq \operatorname{Hom}_{\mathcal{D}}\left(\mathrm{P}\left(\bar{\mu}^{*}\right)\left[-l_{\mu}\right], \mathrm{W}\left(\lambda_{V} \cdot \lambda\right)^{*}\left[-i+n_{\text {top }}\right]\right)_{0} \\
& \simeq \operatorname{Hom}_{\mathcal{D}}\left(\mathrm{P}\left(\bar{\mu}^{*}\right)\left[i-n_{\text {top }}-l_{\mu}\right], \mathrm{W}\left(\lambda_{V} \cdot \lambda\right)^{*}\right)_{0}
\end{aligned}
$$

the first one is the Frobenius reciprocity and the third one is by Lemma 8 (vi). Thus, by (31), Theorem 10 and Corollary 11, we have that

$$
t^{-n_{\mathrm{top}}-l_{\mu}} p_{\mathrm{P}(\mu), \mathrm{M}(\lambda)}=p_{\mathrm{W}\left(\lambda_{V} \cdot \lambda\right)^{*}, \mathrm{~L}\left(\bar{\mu}^{*}\right)}=p_{\mathrm{M}\left(\left(\lambda_{V} \cdot \lambda\right)^{*}\right), \mathrm{L}\left(\bar{\mu}^{*}\right)}=t^{-l_{\mu}-n_{\mathrm{top}}} \overline{p_{\mathrm{M}(\lambda), \mathrm{L}(\mu)}}
$$

and the corollary follows.

\section{Standard filtrations and the BGG Reciprocity}

We say that a (graded) module $\mathrm{N}$ has a (graded) standard filtration, so-called Verma flag, if there exists a sequence of (graded) submodules $0=\mathrm{N}_{0} \subset \mathrm{N}_{1} \subset \cdots \subset$ $\mathrm{N}_{n}=\mathrm{N}$ such that each subquotient $\mathrm{N}_{i} / \mathrm{N}_{i-1}$ is isomorphic to a Verma module. The multiplicity of a Verma module $M(\lambda)$ in $N$ is

$$
[\mathrm{N}: \mathrm{M}(\lambda)]=\#\left\{i \mid \mathrm{N}_{i} / \mathrm{N}_{i-1} \simeq \mathrm{M}(\lambda)\right\} .
$$

We also define $[\mathrm{N}: \mathrm{M}(\lambda)[\ell]]=\#\left\{i \mid \mathrm{N}_{i} / \mathrm{N}_{i-1} \simeq \mathrm{M}(\lambda)[\ell]\right\}$ for the graded case.

The following lemmas are analogous to [19, Thm. 3.6 and Prop. 3.7 (b)].

Lemma 13. Let $\lambda$ be a weight and $\mathrm{N}$ a (graded) module. Then $\mathrm{M}(\lambda) \otimes \mathrm{N}$ has a (graded) standard filtration.

Proof. Let $0=N_{0} \subset N_{1} \subset \cdots \subset N_{r}=\lambda \otimes \mathrm{N}$ be a filtration of $\lambda \otimes \mathrm{N}$ by (graded) $\mathcal{D}^{\geq 0}$-modules such that $N_{i} / N_{i-1}$ is a highest-weight $\mu_{i}$, i.e., a Jordan-Hölder series as (graded) $\mathcal{D}^{\geq 0}$-module. Applying the exact functor $\mathcal{D} \otimes_{\mathcal{D} \geq 0}(-)$ we obtain the desired filtration on $\mathrm{M}(\lambda) \otimes \mathrm{N}$ using Lemma 5 with $U=\lambda$.

Lemma 14. Let $\mathrm{N}$ be a (graded) module which has a (graded) standard filtration and $\mathrm{N}=\mathrm{N}^{\prime} \oplus \mathrm{N}^{\prime \prime}$ as (graded) modules. Then $\mathrm{N}^{\prime}$ and $\mathrm{N}^{\prime \prime}$ have (graded) standard filtrations. 


\section{CRISTIAN VAY}

Proof. By the (graded) standard filtration of $\mathrm{N}$ we have an inclusion of $\iota: \mathrm{M}(\lambda) \rightarrow$ $\mathrm{N}$. Let $p^{\prime}: \mathrm{N} \rightarrow \mathrm{N}^{\prime}$ be the natural projection and $\iota^{\prime}$ its section. We can assume that $\left(p^{\prime} \circ \iota\right)(1 \otimes \lambda) \neq 0$ since the Verma modules have simple socle. Therefore $\left(\iota^{\prime} \circ p^{\prime} \circ \iota\right): \mathrm{M}(\lambda) \rightarrow \mathrm{N}^{\prime}$ is an inclusion and the lemma follows by induction since $\mathrm{N} / \mathrm{M}(\lambda)=\mathrm{N}^{\prime} / \mathrm{M}(\lambda) \oplus \mathrm{N}^{\prime \prime}$.

The main result of the section is the following, cf. [19, Thms. 3.10 and 3.11]. Recall the definition of $a_{\mathrm{P}, \mathrm{M}(\lambda), i}$ from (31).

Theorem 15. Every projective module $\mathrm{P}$ has a graded standard filtration and

$$
[\mathrm{P}: \mathrm{M}(\lambda)[i]]=a_{\mathrm{P}, \mathrm{M}(\lambda), i}
$$

holds for all $\lambda \in \Lambda$. Therefore the BGG Reciprocity

$$
[\mathrm{P}(\mu): \mathrm{M}(\lambda)]=[\mathrm{M}(\lambda): \mathrm{L}(\mu)]
$$

holds for all $\lambda, \mu \in \Lambda$.

Proof. By Lemma 4 and Lemma 13, $\operatorname{lnd}(\lambda) \simeq \mathrm{M}(\lambda) \otimes \mathrm{W}(\varepsilon)$ has a graded standard filtration. The indecomposable projective $\mathrm{P}(\lambda)$ is a graded direct summand of $\operatorname{lnd}(\lambda)$ by (17) and the above section. Hence it has a graded standard filtration by Lemma 14. Therefore all the projective modules have a standard filtration.

The equality $[\mathrm{P}: \mathrm{M}(\lambda)[i]]=a_{\mathrm{P}, \mathrm{M}(\lambda), i}$ is clear and therefore the BGG Reciprocity follows from Corollary 12 by evaluating the polynomials at $t=t^{-1}=1$.

We point out the information about the structure of the indecomposable projective modules which can be deduced from the above results.

Remark 4. Let $0=\mathrm{N}_{0} \subset \mathrm{N}_{1} \subset \cdots \subset \mathrm{N}_{n}=\mathrm{P}(\lambda)$ be a graded standard filtration of $\mathrm{P}(\lambda)$ whose subquotients are $\mathrm{N}_{i} / \mathrm{N}_{i-1} \simeq \mathrm{M}\left(\lambda_{i}\right)\left[\ell_{i}\right]$ for all $i=1, \ldots n$.

Hence, $\mathrm{P}(\lambda) \simeq \mathrm{M}\left(\lambda_{1}\right)\left[\ell_{1}\right] \oplus \cdots \oplus \mathrm{M}\left(\lambda_{n}\right)\left[\ell_{n}\right]$ as $\mathbb{Z}$-graded $\mathcal{D} \leq 0$-modules.

Via this isomorphism, $\bar{V} \cdot\left(1 \otimes \lambda_{i}\right)\left[\ell_{i}\right] \subseteq\left(1 \otimes \lambda_{i-1}\right)\left[\ell_{i}+1\right]$. Moreover, if we know this action, we can infer inductively the action of $\bar{V}$ on $\mathrm{M}\left(\lambda_{i}\right)\left[\ell_{i}\right]$ using the commutation rules between $V$ and $\bar{V}$, cf. [28, p. 438].

By Lemma $8, \mathrm{M}\left(\lambda_{1}\right)\left[\ell_{1}\right]=\mathrm{M}\left(\lambda_{\bar{V}} \cdot \bar{\lambda}\right)\left[l_{\lambda}+n_{\text {top }}\right]$ and $\mathrm{M}\left(\lambda_{n}\right)\left[\ell_{n}\right]=\mathrm{M}(\lambda)$.

\subsection{Simple and projective Verma modules}

The next corollary is a direct consequence of the BGG Reciprocity. However, we give another nice proof without using the former theorem.

Lemma 16. Let $f: \mathrm{N} \rightarrow \mathrm{M}(\lambda)$ be a projection. If $S \subset \mathrm{N}$ is a highest-weight such that $f(S)=1 \otimes \lambda$, then $f$ splits.

Proof. We define a morphism $\phi: \mathrm{M}(\lambda) \rightarrow \mathrm{N}$ by $\phi(1 \otimes \lambda)=S$ and hence $f \circ \phi=\mathrm{id}_{\mathrm{M}}$.

Corollary 17. Let $\mathrm{M}(\lambda)$ be a Verma module. The following are equivalent:

(i) $\mathrm{M}(\lambda)$ is simple.

(ii) $\mathrm{M}(\lambda)$ is projective.

(iii) $\mathrm{M}(\lambda)$ is injective. 


\section{ON PROJECTIVE MODULES OVER FINITE QUANTUM GROUPS}

Proof. Since $\mathcal{D}$ is a finite-dimensional Hopf algebra, a $\mathcal{D}$-module is projective if and only if it is injective. Then (ii) and (iii) are equivalent.

Assume that $\mathrm{M}=\mathrm{M}(\lambda)$ is simple. We shall prove that every projection $f$ : $\mathrm{N} \rightarrow \mathrm{M}$ splits. By Lemma 16, it is enough to find a highest-weight $S \subset \mathrm{N}$ such that $f(S)=1 \otimes \lambda$. Let $S^{\prime}$ be a weight of $\mathrm{N}$ such that $f\left(S^{\prime}\right)=\mathfrak{B}^{n_{\mathrm{top}}}(V) \otimes \lambda$. Then $S=y_{\text {top }} S^{\prime}$ is a highest-weight of $\mathrm{N}$ and $f\left(y_{\text {top }} S^{\prime}\right)=1 \otimes \lambda$ by [28, Cor. 15]. Hence (i) implies (ii).

Assume now that $\mathrm{M}=\mathrm{M}(\lambda)$ is projective. Let $f: \operatorname{lnd}(\lambda) \rightarrow \mathrm{M}$ be the projection such that $f(\lambda)=1 \otimes \lambda$ and $\phi: \mathrm{M} \rightarrow \operatorname{Ind}(\lambda)$ a section of $f$. Hence $\phi(1 \otimes \lambda) \subset$ $y_{\text {top }} \otimes \mathfrak{B}(V) \otimes \lambda$ since $\bar{V} S=0$. That is, for each $s \in S$ there exists $x_{s} \in \mathfrak{B}(V)$ such that $\phi(s)=y_{\text {top }} \otimes x_{s} \otimes s$ and then $s=f \phi(s)=y_{\text {top }} x_{s} s$. As the Verma module is graded, $x_{s} \in \mathfrak{B}^{n_{\mathrm{top}}}(V)$ and is non-zero if $s \neq 0$. Therefore $\mathrm{M}$ is simple by [28, Cor. 15]. Then (ii) implies (i).

We obtain a useful result if we combine the above corollary and the characterization of the simple modules $\mathrm{L}(\lambda)$ and $\mathrm{S}(\lambda)$.

Corollary 18. Let $\mathrm{N}$ be a module and $\mathrm{M}(\lambda)$ a simple Verma module. Assume that either $\lambda$ is a highest-weight of $\mathrm{N}$ or $\lambda_{V} \cdot \lambda$ is a lowest-weight of $\mathrm{N}$. Then $\mathrm{M}(\lambda)$ is a direct summand of $\mathrm{N}$.

Proof. If $\lambda$ is a highest-weight of $\mathrm{N}$, then we have a non-trivial morphism $f$ : $\mathrm{M}(\lambda) \rightarrow \mathrm{N}$. As $\mathrm{M}(\lambda)$ is simple, $f$ is a monomorphism. But also $\mathrm{M}(\lambda)$ is injective, then $f$ splits.

Assume that $\lambda_{V} \cdot \lambda$ is a lowest-weight. Let $\mathrm{U}$ be a simple quotient of the submodule generated by $\lambda_{V} \cdot \lambda$. As there is a unique simple lowest-weight module of weight $\lambda_{V} \cdot \lambda, \mathrm{U}=\mathrm{S}(\lambda)=\mathrm{M}(\lambda)$. Then $\mathrm{M}(\lambda)$ is a direct summand of $\mathrm{U}$ and hence it is of $\mathrm{N}$ because $\mathrm{M}(\lambda)$ is injective.

\subsection{Co-standard filtration}

We say that a (graded) module has a (graded) co-standard filtration if it is filtered by (graded) modules whose subquotients are isomorphic to co-Verma modules $\mathbf{W}(\lambda)$, recall $(13)$. The multiplicities $[N: \mathbf{W}(\lambda)]$ and $[N: W(\lambda)[\ell]]$ are defined as for standard filtrations. We next formulate analogous results to those about standard filtrations. The proofs are similar.

Lemma 19. Let $\lambda$ be a weight and $\mathrm{N}$ a (graded) module.

(i) Then $\mathrm{M}(\lambda) \otimes \mathrm{N}$ has a (graded) standard filtration.

(ii) If $\mathrm{N}$ has a (graded) standard filtration and $\mathrm{N}=\mathrm{N}^{\prime} \oplus \mathrm{N}^{\prime \prime}$ as (graded) modules, then $\mathrm{N}^{\prime}$ and $\mathrm{N}^{\prime \prime}$ have (graded) standard filtrations.

Let $\mathrm{P}$ be a graded projective module. Then, it is also graded projective as a $\mathcal{D}^{\geq 0}$-module. For each $\lambda \in \Lambda$ and $i \in \mathbb{Z}$, we define

$$
\begin{aligned}
a_{\mathrm{P}, \mathrm{W}(\lambda), i} & =\operatorname{dim} \operatorname{Hom}_{\mathcal{D} \geq 0}\left(\lambda_{\bar{V}} \cdot \lambda, \mathrm{P}\right)_{i+n_{\mathrm{top}}} \quad \text { and } \\
p_{\mathrm{P}, \mathrm{W}(\lambda)} & =\sum_{i} a_{\mathrm{P}, \mathrm{W}(\lambda), i} t^{i} \in \mathbb{Z}\left[t, t^{-1}\right] ;
\end{aligned}
$$




\section{CRISTIAN VAY}

we assume that the highest-weight $\lambda_{\bar{V}} \cdot \lambda$ is concentrated in degree 0 . Therefore

$$
\mathrm{P} \simeq \bigoplus_{\lambda \in \Lambda} p_{\mathrm{P}, \mathrm{W}(\lambda)} \cdot \mathrm{W}(\lambda)
$$

as $\mathcal{D}^{\geq 0}$-modules.

The second item below is the BGG Reciprocity for co-Verma modules. Item (iii) says that the multiplicities of the co-Verma modules can be deduced from the composition factors of the Verma modules.

Theorem 20. Every projective module $\mathrm{P}$ has a graded co-standard filtration and the following identities hold for all $\lambda, \mu \in \Lambda$ :

(i) $[\mathrm{P}: \mathrm{W}(\lambda)[i]]=a_{\mathrm{P}, \mathrm{W}(\lambda), i}$.

(ii) $p_{\mathrm{P}(\mu), \mathrm{W}(\lambda)}=\overline{p_{\mathrm{W}(\lambda), \mathrm{L}(\mu)}}$ and $[\mathrm{P}(\mu): \mathrm{W}(\lambda)]=[\mathrm{W}(\lambda): \mathrm{L}(\mu)]$.

(iii) $p_{\mathrm{P}(\mu), \mathrm{W}(\lambda)}=t^{-n_{\text {top }}} \overline{p_{\mathrm{M}\left(\lambda_{\bar{V}} \cdot \lambda\right), \mathrm{L}(\mu)}}$ and $[\mathrm{P}(\mu): \mathrm{W}(\lambda)]=\left[\mathrm{M}\left(\lambda_{\bar{V}} \cdot \lambda\right): \mathrm{L}(\mu)\right]$.

\subsection{Tensor product of projective modules}

Theorem 21. Let $\mathrm{P}$ and $\mathrm{Q}$ be projective modules. Then

$$
\mathrm{P} \otimes \mathrm{Q} \simeq \bigoplus_{\lambda, \mu \in \Lambda} p_{\mathrm{P}, \mathrm{W}(\lambda)} p_{\mathrm{Q}, \mathrm{M}(\mu)} \operatorname{lnd}(\lambda \cdot \mu) .
$$

Proof. Let $0=\mathrm{Q}_{0} \subset \mathrm{Q}_{1} \subset \cdots \subset \mathrm{Q}_{n}=\mathrm{Q}$ be a graded standard filtration of $\mathrm{Q}$. Since $P \otimes-$ is exact and $P \otimes N$ is projective for any module, we have that

$$
\mathrm{P} \otimes \mathrm{Q} \simeq \bigoplus_{i=1}^{n} \mathrm{P} \otimes\left(\mathrm{Q}_{i} / \mathrm{Q}_{i-1}\right) \simeq \bigoplus_{\mu \in \Lambda} p_{\mathrm{Q}, \mathrm{M}(\mu)} \mathrm{P} \otimes \mathrm{M}(\mu)
$$

Let $0=\mathrm{P}_{0} \subset \mathrm{P}_{1} \subset \cdots \subset \mathrm{P}_{n}=\mathrm{P}$ be a graded co-standard filtration of $\mathrm{P}$. By Lemma $4, \mathrm{~W}(\lambda) \otimes \mathrm{M}(\mu) \simeq \operatorname{Ind}(\lambda \cdot \mu)$ is projective. Then

$$
\mathrm{P} \otimes \mathrm{M}(\mu) \simeq \bigoplus_{i=1}^{n}\left(\mathrm{P}_{i} / \mathrm{P}_{i-1}\right) \otimes \mathrm{M}(\mu) \simeq \bigoplus_{\lambda \in \Lambda} p_{\mathrm{P}, \mathrm{W}(\lambda)} \operatorname{lnd}(\lambda \cdot \mu)
$$

and the theorem follows.

\section{Examples}

\subsection{Taft algebras}

Let $G=C_{n}=\langle g\rangle$ be the cyclic group of order $n$ and $q$ a $n$-th primitive root of unity. The quantum line $\mathbb{k}\left\langle x \mid x^{n}=0\right\rangle$ is isomorphic to the Nichols algebra of $V=\mathbb{k} x \in \mathbb{k}_{\mathbb{k} G} \mathcal{Y} \mathcal{D}$ with action $g \cdot x=q x$ and coaction $\rho(x)=g \otimes x$. The Taft algebra $T_{q}$ is the bosonization $\mathfrak{B}(V) \# \mathbb{k} G$. The Frobenius-Lusztig kernel $\mathfrak{u}_{q}(\mathfrak{s l}(2))$ is isomorphic to a quotient of the Drinfeld double $\mathcal{D}\left(T_{q}\right)$ by a central group-like element. The simple modules of $\mathcal{D}\left(T_{q}\right)$ and $\mathfrak{u}_{q}(\mathfrak{s l}(2))$ were studied for instance in [12] and [1], [11], [15], [32], respectively.

In the case of $\mathcal{D}\left(T_{q}\right), \Lambda \simeq C_{n} \times C_{n}=\left\langle\chi_{1}\right\rangle \times\left\langle\chi_{2}\right\rangle$ and all the Verma modules $\mathrm{M}(r, s)=\mathrm{M}\left(\chi_{1}^{r}, \chi_{2}^{s}\right)$ have dimension $n$. The simple module $\mathrm{L}(r, 1-(r+l))$ has dimension $l$ for $1 \leq l, r \leq n$ [12, Thm. 2.5]. Therefore the Verma modules $\mathrm{M}(r, 1-$ $(r+n))$ are simple and projective by Corollary 17 .

It is easy to see that the composition factors of $\mathrm{M}(r, 1-(r+l))$ are $\mathrm{L}(r, 1-(r+$ $l)$ ) and $\mathrm{L}(r+l, 1-((r+l)+(n-l)))$. Therefore the indecomposable projective $\mathrm{P}(r, 1-(r+l))$ has a submodule $\mathrm{N} \simeq \mathrm{M}(r+l-n, 1-((r+l-n)+(n-l)))$ and $\mathrm{P}(r, 1-(r+l)) / \mathrm{N} \simeq \mathrm{M}(r, 1-(r+l))$ by the BGG Reciprocity. Notice that the module in [12, Remark 2.8] is $\mathrm{M}(r, 1-(r+n-1))$.

The previous facts do not appear in [12]. Of course, we can also come to the same conclusion using the knowledge about modules over $\mathfrak{u}_{q}(\mathfrak{s l}(2))$. 


\subsection{The Shapovalov determinant}

Assume $\mathfrak{B}(V)$ is a Nichols algebra of diagonal type. In [18] the authors give a formula analogous to the Shapovalov determinant for complex semisimple Lie algebras. Thus, they characterize the simple Verma modules of the Drinfeld doubles attached to $\mathfrak{B}(V)$. We now know that this also gives a characterization of the projective Verma modules by Corollary 17.

\subsection{The Nichols algebra of unidentified diagonal type $\mathfrak{u} \mathfrak{f} \mathfrak{o}(7)$}

This is the smallest Nichols algebra $\mathfrak{B}(V)$ of unidentified type, $\operatorname{dim} \mathfrak{B}(V)=144$, see [7]. Let $G$ be an abelian group such that $\mathfrak{B}(V) \in \mathbb{k}_{\mathbb{k} G} \mathcal{Y} \mathcal{D}$ and $\mathcal{D}$ the Drinfeld double of $\mathfrak{B}(V) \# \mathbb{k} G$. The simple modules of $\mathcal{D}$ are classified in [2]. The authors divide the set of weights in 47 subsets and study the corresponding Verma modules case by case. They obtain three families of weights $\left(\mathcal{C}_{0}, \mathcal{C}_{1}, \mathcal{C}_{2}\right)$ which are related with the Shapovalov determinant.

They use the Shapovalov determinant to show that the Verma modules in $\mathcal{C}_{0}$ are simple [2, Lem. 1.6]. Hence these are projective by Corollary 17.

The class $\mathcal{C}_{1}$ is formed by 9 different types of weights. The composition factors of the Verma module $\mathrm{M}(\lambda), \lambda \in \mathcal{C}_{1}$, are given explicitly in [2, Sect. 2]. These are $\mathrm{L}(\lambda)$ and $\mathrm{L}\left(\lambda^{\prime}\right)$ for certain $\lambda^{\prime} \in \mathcal{C}_{1}$. Then, using the BGG Reciprocity, we deduce that the projective cover $\mathrm{P}(\lambda)$ of $\mathrm{L}(\lambda)$ has a submodule isomorphic to $\mathrm{M}(\mu)$ for certain $\mu \in \mathcal{C}_{1}$ satisfying $\mu^{\prime}=\lambda$ and $\mathrm{P}(\lambda) / \mathrm{M}(\mu) \simeq \mathrm{M}(\lambda)$.

The Verma module in $\mathcal{C}_{2}$ might have more than two composition factors, see $[2$, Rem. 3.2]. It is possible to obtain the composition factors of $M(\lambda)$, for all $\lambda \in \mathcal{C}_{2}$, by reasoning as in [2, Rem. 3.2]; the graded characters can also help.

\subsection{The Fomin-Kirillov algebra $\mathcal{F} \mathcal{K}_{3}$}

This is a Nichols algebra in $\mathbb{R S}_{3} \mathcal{V} \mathcal{D}$, it is the smallest one over a non-abelian group. In [28] we investigate the Verma and simple modules over the Drinfeld double of $\mathcal{F} \mathcal{K}_{3} \# \mathbb{k}_{3}$. In this case, the set of weights is

$$
\Lambda=\{(e,+),(e,-),(e, \rho),(\sigma,+),(\sigma,-),(\tau, 0),(\tau, 1),(\tau, 2)\}
$$

We have shown that $\mathrm{M}(e,-), \mathrm{M}(\tau, 1), \mathrm{M}(\tau, 2)$ and $\mathrm{M}(\sigma,+)$ are simple. Therefore they are projective by Corollary 17 . The composition factors of the remaining Verma modules are given in [28, Thms. 7, 8, 9 and 10]. We have that

- $\operatorname{ch} \mathrm{M}(\sigma,-)=2 \cdot \operatorname{ch} \mathrm{L}(\sigma,-)+2 \cdot \operatorname{ch} \mathrm{L}(e,+)+\operatorname{ch} \mathrm{L}(\tau, 0)+\operatorname{ch} \mathrm{L}(e, \rho)$.

- $\operatorname{ch} \mathrm{M}(e,+)=2 \cdot \operatorname{ch} \mathrm{L}(e,+)+\operatorname{ch} \mathrm{L}(\sigma,-)$.

- $\operatorname{ch} \mathrm{M}(e, \rho)=\operatorname{ch} \mathrm{M}(\tau, 0)=\operatorname{ch} \mathrm{L}(\tau, 0)+\operatorname{ch} \mathrm{L}(e, \rho)+\operatorname{ch} \mathrm{L}(\sigma,-)$.

By the BGG Reciprocity, we conclude that

- $\operatorname{ch} \mathrm{P}(\sigma,-)=2 \cdot \operatorname{ch} \mathrm{M}(\sigma,-)+\operatorname{ch} \mathrm{M}(e,+)+\operatorname{ch} \mathrm{M}(\tau, 0)+\operatorname{ch} \mathrm{M}(e, \rho)$.

- $\operatorname{ch} \mathrm{P}(e,+)=2 \cdot \operatorname{ch} \mathrm{M}(e,+)+2 \cdot \operatorname{ch} \mathrm{M}(\sigma,-)$.

- $\operatorname{ch} \mathrm{P}(e, \rho)=\operatorname{ch} \mathrm{M}(\tau, 0)=\operatorname{ch} \mathrm{M}(\tau, 0)+\operatorname{ch} \mathrm{M}(e, \rho)+\operatorname{ch} \mathrm{M}(\sigma,-)$.

Together with Barbara Pogorelsky, we study these projective modules in more detail [29]. We also discuss the tensor product between the simple and projective modules. 


\section{CRISTIAN VAY}

\section{References}

[1] H. H. Andersen, J. C. Jantzen, W. Soergel, Representations of quantum groups at a pth root of unity and of semisimple groups in characteristic $p$ : independence of $p$, Astérisque 220 (1994), 1-321.

[2] N. Andruskiewitsch, I. Angiono, A. Mejía, C. Renz, Simple modules of the quantum double of the Nichols algebra of unidentified diagonal type $\mathfrak{u f o}(7)$, Commun. Algebra (2017), http://dx.doi.org/10.1080/00927872.2017.1357726.

[3] N. Andruskiewitsch, M. Graña, Braided Hopf algebras over non-abelian finite groups, Bol. Acad. Nac. Cienc. (Córdoba) 63 (1999), 45-78.

[4] N. Andruskiewitsch, I. Heckenberger, H.-J. Schneider, The Nichols algebra of a semisimple Yetter-Drinfeld module, Amer. J. Math. 132 (2010), 1493-1547.

[5] N. Andruskiewitsch, D. Radford, H.-J. Schneider, Complete reducibility theorems for modules over pointed Hopf algebras, J. Algebra 324 (2010), 2932-2970.

[6] N. Andruskiewitsch, H.-J. Schneider, Pointed Hopf algebras, in: New Directions in Hopf Algebras, Math. Sci. Res. Inst. Publ., Vol. 43, Cambridge Univ. Press, Cambridge, 2002, pp. 1-68.

[7] I. Angiono, Nichols algebras of unidentified diagonal type, Comm. Algebra 41 (2013), 4667-4693.

[8] S. Azam, H. Yamane, M. Yousofzadeh, Classification of finite-dimensional irreducible representations of generalized quantum groups via Weyl groupoids, Publ. Res. Inst. Math. Sci. 51 (2015), 59-130.

[9] G. Bellamy, U. Thiel, Highest weight theory for finite-dimensional graded algebras with triangular decomposition, arXiv:1705.08024 (2017).

[10] И. Н. Бернштейн, И. М. Гельфанд, С. И. Гельфанд, Об одной категории gмодулей, Функц. анализ и его прил. 10 (1976), вып. 2, 1-8. Engl transl.: I. N. Bernstein, I. M. Gel'fand, S. I. Gel'fand, Category of $\mathfrak{g}$-modules, Funct. Analysis and Its Appl. 10 (1976), no. 2, 87-92.

[11] V. Chari, A. Premet, Indecomposable restricted representations of quantum $\mathfrak{s l}_{2}$, Publ. Res. Inst. Math. Sci. 30 (1994), 335-352.

[12] H.-X. Chen, Irreducible representations of a class of quantum doubles, J. Algebra 225 (2000), 391-409.

[13] E. Cline, B. Parshall, L. Scott, Finite-dimensional algebras and highest weight categories, J. Reine Angew. Math. 391 (1988), 85-99.

[14] C. W. Curtis, I. Reiner, Methods of Representation Theory. Vol. I, With Applications to Finite Groups and Orders, Wiley Classics Library, A Wiley-Interscience Publication, John Wiley \& Sons, New York, 1990.

[15] C. M. Drupieski, Representations and cohomology for Frobenius-Lusztig kernels, J. Pure Appl. Algebra 215 (2011), 1473-1491.

[16] H. Garland, J. Lepowsky, Lie algebra homology and the Macdonald-Kac formulas, Invent. Math. 34 (1976), no. 1, 37-76.

[17] R. Gordon, E. L. Green, Graded Artin algebras, J. Algebra 76 (1982), 111-137.

[18] I. Heckenberger, H. Yamane, Drinfeld doubles and Shapovalov determinants, Rev. Un. Mat. Argentina 51 (2010), 107-146. 


\section{ON PROJECTIVE MODULES OVER FINITE QUANTUM GROUPS}

[19] J. E. Humphreys, Representations of Semisimple Lie Algebras in the BGG Category $\mathcal{O}$, Graduate Studies in Mathematics, Vol. 94, American Mathematical Society, Providence, RI, 2008.

[20] R. S. Irving, Projective modules in the category $\mathcal{O}$, unpublished manuscript, 1982.

[21] R. S. Irving, BGG algebras and the BGG reciprocity principle, J. Algebra 135 (1990), 363-380.

[22] A. S. Kleshchev, Affine highest weight categories and affine quasihereditary algebras, Proc. Lond. Math. Soc. Third Series 110 (2015), 841-882.

[23] L. Krop, D. E. Radford, Simple modules for the Drinfeld double of a class of Hopf algebras, in: Groups, Rings and Algebras, Contemp. Math., Vol. 420, Amer. Math. Soc., Providence, RI, 2006, pp. 229-235.

[24] L. Krop, D. E. Radford, Representations of pointed Hopf algebras and their Drinfeld quantum doubles, J. Algebra 321 (2009), 2567-2603.

[25] M. Lorenz, Representations of finite-dimensional Hopf algebras, J. Algebra 188 (1997), 476-505.

[26] G. Lusztig, Leading coefficients of character values of Hecke algebras, in: The Arcata Conference on Representations of Finite Groups (Arcata, Calif., 1986), Proc. Sympos. Pure Math., Vol. 47, Amer. Math. Soc., Providence, RI, 1987, pp. 235-262.

[27] U. Oberst, H.-J. Schneider, Über Untergruppen endlicher algebraischer Gruppen, Manuscripta Math. 8 (1973), 217-241.

[28] B. Pogorelsky, C. Vay, Verma and simple modules for quantum groups at non-abelian groups, Adv. Math. 301 (2016), 423-457.

[29] B. Pogorelsky, C. Vay, On the representation theory of a quantum group attached to the Fomin-Kirillov algebra $\mathcal{F K}_{3}$, arXiv: 1707.02091 (2017).

[30] D. E. Radford, Minimal quasitriangular Hopf algebras, J. Algebra 157 (1993), 285315.

[31] D. E. Radford, H.-J. Schneider, On the simple representations of generalized quantum groups and quantum doubles, J. Algebra 319 (2008), 3689-3731.

[32] R. Suter, Modules over $\mathfrak{U}_{q}\left(\mathfrak{s l}_{2}\right)$, Comm. Math. Phys. 163 (1994), 359-393.

[33] S. J. Witherspoon, The representation ring of the quantum double of a finite group, J. Algebra 179 (1996), 305-329. 